\title{
LIMIT THEOREMS FOR PARTIAL SUM PROCESSES OF A GAUSSIAN SEQUENCE
}

\author{
Yong-KaB Choi, KYO-SHin HWAng, \\ HeE-Jin Moon, TAE-Sung Kim And Jong-Il BAEK
}

A BSTRACT. In this paper we establish limsup and liminf theorems for the increments of partial sum processes of a dependent stationary Gaussian sequence.

\section{Introduction and results}

Let $\left\{X_{j} ; j=1,2, \ldots\right\}$ be a sequence of independent identically distributed (i.i.d) random variables and let $\mathbf{S}_{0}=0$ and $\mathbf{S}_{n}=\sum_{j=1}^{n} X_{j}$. For an integer sequence $\left\{a_{n} ; n=1,2, \ldots\right\}$ with $1 \leq a_{n} \leq n$, put

$$
U_{n}=\max _{1 \leq k \leq n-a_{n}}\left(\boldsymbol{S}_{k+a_{n}}-\boldsymbol{S}_{k}\right) .
$$

Csörgő and Révész [6] obtained the following strong limit law

$$
\lim _{n \rightarrow \infty} \frac{U_{n}}{b_{n}}=1 \quad \text { a.s. }
$$

under some conditions of $\left\{X_{j}\right\}$ and $\left\{a_{n}\right\}$, where $\left\{b_{n} ; n=1,2, \ldots\right\}$ is some sequence of constants. For further various results on this limit law (1.1) about the sequence of i.i.d. random variables, we refer to ([5], [7], [8], [9], [11], [19], [20], [21]).

On the other hand, Lin ([15], [16], [18]) established large increment results for a sequence of independent or mixing dependent random variables. Theoretically and practically, strong dependent sequences are

Received September 3, 2003.

2000 Mathematics Subject Classification: 60F10, 60F15, 60G15.

Key words and phrases: Gaussian sequence, large deviation probability, regularly varying function.

This work was supported by KRF Grant (2002-042-D00008). 
important and interesting. Usually one considers the case of Gaussian sequences.

Horvàth and Shao [10] studied extreme value limit distributions for the maximum of partial sums of a stationary Gaussian sequence with long-range dependence.

Recently, Csáki and Gonchigdanzan [4] investigated almost sure central limit theorems for the maximum of dependent stationary Gaussian sequences.

In this paper we are interested in the strong limit law types as in (1.1) about partial sum processes of a dependent stationary Gaussian sequence. Let $\left\{\xi_{j} ; j=1,2, \ldots\right\}$ be a centered stationary Gaussian sequence with $E \xi_{1}^{2}=1$ and $\rho_{n}=E\left(\xi_{1} \xi_{1+n}\right), n \geq 1$. Put $S_{0}=0$, $S_{n}=\sum_{j=1}^{n} \xi_{j}$ and $\sigma(n)=\sqrt{E S_{n}^{2}}$. Assume that $\sigma(n)$ can be extended to a continuous function $\sigma(t)$ of $t>0$ which is nondecreasing and regularly varying with exponent $\alpha$ for some $0<\alpha<1$. Suppose that $\left\{a_{n} ; n \geq 1\right\}$ is a sequence of positive integers such that

(i) $1 \leq a_{n} \leq n$.

Denote $\beta_{n}=\left\{2\left(\log \left(n / a_{n}\right)+\log \log n\right)\right\}^{1 / 2}$ for $n>e$.

Recently, Choi et al. [3] proved the following Theorems A and B.

THEOREM A. Suppose that the sequence $\left\{a_{n} ; n \geq 1\right\}$ satisfies conditions (i) and

(ii) $\limsup a_{n} / n=: \mu<1$,

(iii) there exist $0<\mu_{2} \leq \mu_{1} \leq 1$ such that, for any $m<n$, we have $\mu_{1} a_{m} \leq a_{n}$ and $\mu_{2} a_{m} / m \geq a_{n} / n$.

Assume that, for $n \geq 1$, either

or

(iv) $\rho_{n} \leq 0$

(v) $\left|\rho_{n}\right| \leq \sigma^{2}(n) / n^{2}$.

Then we have

$$
\begin{aligned}
& \limsup _{n \rightarrow \infty} \sup _{0 \leq i \leq n} \sup _{1 \leq j \leq a_{n}} \frac{\left|S_{i+j}-S_{i}\right|}{\sigma\left(a_{n}\right) \beta_{n}}=1 \quad \text { a.s., } \\
& \underset{n \rightarrow \infty}{\limsup } \frac{\left|S_{n+a_{n}}-S_{n}\right|}{\sigma\left(a_{n}\right) \beta_{n}}=1 \quad \text { a.s. }
\end{aligned}
$$

Next, consider the case of a limit result. 
THEOREM B. Suppose that the condition (i) and one of (iv) and (v) are satisfied. Further suppose that

(vi) $\lim _{n \rightarrow \infty} \frac{\log \left(n / a_{n}\right)}{\log \log n}=\infty$.

Then we have

$$
\begin{aligned}
& \lim _{n \rightarrow \infty} \sup _{0 \leq i \leq n} \sup _{1 \leq j \leq a_{n}} \frac{\left|S_{i+j}-S_{i}\right|}{\sigma\left(a_{n}\right) \beta_{n}}=1 \quad \text { a.s. } \\
& \lim _{n \rightarrow \infty} \sup _{0 \leq i \leq n} \frac{\left|S_{i+a_{n}}-S_{i}\right|}{\sigma\left(a_{n}\right) \beta_{n}}=1 \quad \text { a.s. }
\end{aligned}
$$

Note that the condition (v) implies that, for $n \geq 1$,

$$
-n^{2 \alpha-2} L(n) \leq \rho_{n} \leq n^{2 \alpha-2} L(n),
$$

where $L(n)$ is a slowly varying function.

For the Wiener process $\{W(t), 0 \leq t<\infty\}$ with independent increments, Book and Shore [1] proved that liminf results are different from limsup results if the following condition

$$
\lim _{T \rightarrow \infty} \frac{\log \left(T / a_{T}\right)}{\log \log T}=\infty
$$

of Theorem 1.2.1 in [6] is replaced by

$$
\lim _{T \rightarrow \infty} \frac{\log \left(T / a_{T}\right)}{\log \log T}=r, \quad 0 \leq r<\infty .
$$

On this point of view, the main objective of this paper is to show that liminf results are different from the results (1.2) and (1.3) for dependent Gaussian sequences if the condition (vi) is replaced by

$$
\lim _{n \rightarrow \infty} \frac{\log \left(n / a_{n}\right)}{\log _{\theta} \log n}=r, \quad 0 \leq r<\infty,
$$

where $\theta=1+\varepsilon$ for $\varepsilon>0$ small enough.

The main results are as follows: 
THEOREM 1.1. If the condition (i) and

$$
\lim _{n \rightarrow \infty} \frac{\log \left(n / a_{n}\right)}{\log \log n}=r, \quad 0 \leq r \leq \infty
$$

are satisfied, then we have

$$
\liminf _{n \rightarrow \infty} \sup _{0 \leq i \leq n} \sup _{1 \leq j \leq a_{n}} \frac{\left|S_{i+j}-S_{i}\right|}{\sigma\left(a_{n}\right) \beta_{n}} \leq\left(\frac{r}{1+r}\right)^{1 / 2} \quad \text { a.s. }
$$

The following theorem is straightforward from Theorem 1.1.

THEOREM 1.2. If the condition (i) and

(vii) $^{\prime} \quad \lim _{n \rightarrow \infty} \frac{\log \left(n / a_{n}\right)}{\log _{\theta} \log n}=r, \quad 0 \leq r \leq \infty$

are satisfied, then we have

$$
\liminf _{n \rightarrow \infty} \sup _{0 \leq i \leq n} \sup _{1 \leq j \leq a_{n}} \frac{\left|S_{i+j}-S_{i}\right|}{\sigma\left(a_{n}\right) \beta_{n}^{\prime}} \leq\left(\frac{r}{1+r}\right)^{1 / 2} \quad \text { a.s. }
$$

where $\beta_{n}^{\prime}=\left\{2\left(\log \left(n / a_{n}\right)+\log _{\theta} \log n\right)\right\}^{1 / 2}$ for $n>e$.

THEOREM 1.3. Suppose that conditions (i), (vii)' and one of (iv) and $(\mathrm{v})$ are satisfied. Then we have

$$
\liminf _{n \rightarrow \infty} \sup _{0 \leq i \leq n} \frac{\left|S_{i+a_{n}}-S_{i}\right|}{\sigma\left(a_{n}\right) \beta_{n}^{\prime}} \geq\left(\frac{r}{1+r}\right)^{1 / 2} \quad \text { a.s. }
$$

Combining Theorems 1.2 and 1.3, we obtain the following liminf result.

Corollary 1.1. Under the assumptions of Theorem 1.3, we have

$$
\begin{aligned}
& \liminf _{n \rightarrow \infty} \sup _{0 \leq i \leq n} \sup _{1 \leq j \leq a_{n}} \frac{\left|S_{i+j}-S_{i}\right|}{\sigma\left(a_{n}\right) \beta_{n}^{\prime}}=\left(\frac{r}{1+r}\right)^{1 / 2} \quad \text { a.s., } \\
& \liminf _{n \rightarrow \infty} \sup _{0 \leq i \leq n} \frac{\left|S_{i+a_{n}}-S_{i}\right|}{\sigma\left(a_{n}\right) \beta_{n}^{\prime}}=\left(\frac{r}{1+r}\right)^{1 / 2} \quad \text { a.s. }
\end{aligned}
$$

Note that if $r=\infty$ in (vii)', then (1.3) follows from (1.6) and Theorem 1.1 in Choi et al. [3]; if $0 \leq r<\infty$ in (vii) ${ }^{\prime}$, then (1.7) differs from (1.2) under conditions (ii), (iii) and (vii)'. 


\section{Proofs of main theorems}

The following Lemmas 2.1 and 2.2 are used for the proof of Theorem 1.1, and Lemma 2.1 is an analogue of Lemma 2.2 in [3] (See also Lemma 2.2 in [17]).

Lemma 2.1. For any $\varepsilon>0$, there exists a positive constant $c_{\varepsilon}$ such that

$$
P\left\{\sup _{0 \leq i \leq n} \sup _{1 \leq j \leq a_{n}} \frac{\left|S_{i+j}-S_{i}\right|}{\sigma\left(a_{n}\right)} \geq u\right\} \leq c_{\varepsilon} \frac{n}{a_{n}} \exp \left(-\frac{u^{2}}{2+\varepsilon}\right)
$$

for all $u>1$.

The next Lemma 2.2 is obvious.

Lemma 2.2. Let $\left\{\xi, \xi_{n} ; n \geq 1\right\}$ be a sequence of random variables. If

$$
P\left\{\xi_{n}>\xi\right\} \rightarrow 0 \quad \text { as } n \rightarrow \infty,
$$

then there is a subsequence $\left\{\xi_{n_{k}}\right\}$ such that

$$
\limsup _{k \rightarrow \infty} \xi_{n_{k}} \leq \xi \quad \text { a.s. }
$$

So we have

$$
\liminf _{n \rightarrow \infty} \xi_{n} \leq \xi \quad \text { a.s. }
$$

Proof of Theorem 1.1. First, suppose that $0<r \leq \infty$. From (vii), there exists $\gamma>0$ such that $n / a_{n} \geq(\log n)^{\gamma}$ for sufficiently large $n$. Thus by Lemma 2.1 we have, for any $\varepsilon>0$,

$$
\begin{aligned}
& P\left\{\sup _{0 \leq i \leq n} \sup _{1 \leq j \leq a_{n}} \frac{\left|S_{i+j}-S_{i}\right|}{\sigma\left(a_{n}\right)\left\{2 \log \left(n / a_{n}\right)\right\}^{1 / 2}}>\sqrt{1+\varepsilon}\right\} \\
\leq & c_{\varepsilon} \frac{n}{a_{n}} \exp \left(-\frac{2+2 \varepsilon}{2+\varepsilon} \log \frac{n}{a_{n}}\right) \\
\leq & c_{\varepsilon}(\log n)^{-\gamma \varepsilon /(2+\varepsilon)} \rightarrow 0 \text { as } n \rightarrow \infty .
\end{aligned}
$$

It follows from Lemma 2.2 that

$$
\liminf _{n \rightarrow \infty} \sup _{0 \leq i \leq n} \sup _{1 \leq j \leq a_{n}} \frac{\left|S_{i+j}-S_{i}\right|}{\sigma\left(a_{n}\right)\left\{2 \log \left(n / a_{n}\right)\right\}^{1 / 2}} \leq 1 \quad \text { a.s. }
$$


Hence by (vii) we obtain

$$
\begin{aligned}
& \liminf _{n \rightarrow \infty} \sup _{0 \leq i \leq n} \sup _{1 \leq j \leq a_{n}} \frac{\left|S_{i+j}-S_{i}\right|}{\sigma\left(a_{n}\right) \beta_{n}} \\
= & \liminf _{n \rightarrow \infty} \sup _{0 \leq i \leq n} \sup _{1 \leq j \leq a_{n}} \frac{\left|S_{i+j}-S_{i}\right|}{\sigma\left(a_{n}\right)\left\{2 \log \left(n / a_{n}\right)\right\}^{1 / 2}} \\
\times & \left(\frac{2 \log \left(n / a_{n}\right)}{2\left(\log \left(n / a_{n}\right)+\log \log n\right)}\right)^{1 / 2} \\
\leq & \sqrt{\frac{r}{1+r}} \quad \text { a.s. }
\end{aligned}
$$

On the other hand, consider the case $r=0$. It follows from (vii) that for any small $\varepsilon>0$ we have

$$
\frac{n}{a_{n}}<(\log n)^{\varepsilon /(2+\varepsilon)}
$$

for $n$ sufficiently large. Applying Lemma 2.1 again, we get

$$
\begin{aligned}
& P\left\{\sup _{0 \leq i \leq n} \sup _{1 \leq j \leq a_{n}} \frac{\left|S_{i+j}-S_{i}\right|}{\sigma\left(a_{n}\right) \beta_{n}}>\sqrt{\varepsilon}\right\} \\
\leq & c_{\varepsilon}(\log n)^{-\varepsilon /(2+\varepsilon)} \rightarrow 0 \text { as } n \rightarrow \infty
\end{aligned}
$$

and hence Lemma 2.2 gives

$$
\liminf _{n \rightarrow \infty} \sup _{0 \leq i \leq n} \sup _{1 \leq j \leq a_{n}} \frac{\left|S_{i+j}-S_{i}\right|}{\sigma\left(a_{n}\right) \beta_{n}} \leq 0 \quad \text { a.s. }
$$

Combining (2.1) with (2.2) completes the proof of Theorem 1.1.

The following Lemmas 2.3-2.5 are essential to prove Theorem 1.3.

Lemma 2.3. (cf. Corollary 1.2.2 in [14]) Let $\xi=\left(\xi_{i j}\right)$ and $\eta=\left(\eta_{i j}\right)$, $1 \leq i \leq n, 1 \leq j \leq m$, be centered Gaussian random vectors such that

$$
\begin{aligned}
& E\left(\xi_{i j}^{2}\right)=E\left(\eta_{i j}^{2}\right) \text { for all } i, j \\
& E\left(\xi_{i j} \xi_{i k}\right) \leq E\left(\eta_{i j} \eta_{i k}\right) \text { for all } i, j, k, \\
& E\left(\xi_{i j} \xi_{l k}\right) \geq E\left(\eta_{i j} \eta_{l k}\right) \text { for all } i \neq l, j \text { and } k .
\end{aligned}
$$

Then, for all real numbers $\lambda_{i j}$,

$$
P\left\{\bigcap_{i=1}^{n} \bigcup_{j=1}^{m}\left(\eta_{i j}>\lambda_{i j}\right)\right\} \leq P\left\{\bigcap_{i=1}^{n} \bigcup_{j=1}^{m}\left(\xi_{i j}>\lambda_{i j}\right)\right\} .
$$


Lemma 2.4. ([12], [13]) Let $\xi_{j}(j=1,2, \ldots, n)$ be standardized normal random variables with $\operatorname{Cov}\left(\xi_{i}, \xi_{j}\right)=\Lambda_{i j}$ such that $\delta=\max _{i \neq j}\left|\Lambda_{i j}\right|$ $<1$. Then for any real number $u$ and integers $1 \leq l_{1}<l_{2}<\cdots<l_{k} \leq n$ with $k \leq n$, we have

$$
P\left\{\max _{1 \leq j \leq k} \xi_{l_{j}} \leq u\right\} \leq(\Phi(u))^{k}+c \sum_{1 \leq i<j \leq k}\left|\rho_{i j}\right| \exp \left(-\frac{u^{2}}{1+\left|\rho_{i j}\right|}\right)
$$

where $\rho_{i j}=\Lambda_{l_{i} l_{j}}$ and $c=c(\delta)$ is a constant independent of $n$ and $u$, and $\Phi(u)=\int_{-\infty}^{u} \frac{1}{\sqrt{2 \pi}} \exp \left(-y^{2} / 2\right) d y$.

Under the stationary condition on $\rho_{i j}$, we can estimate an upper bound for the second term of the right hand side of (2.3) as follows:

Lemma 2.5. ([2]) Let $\xi_{j}, \delta, k$ and $\rho_{i j}$ be as in Lemma 2.4. Assume that for some $\nu>0$

$$
\left|\rho_{i j}\right|<|i-j|^{-\nu} \quad \text { for all } i \neq j .
$$

Put $u=\{(2-\eta) \log k\}^{1 / 2}$, where $0<\eta<(1-\delta) \nu /(1+\nu+\delta)$. Then we have

$$
\sum:=\sum_{1 \leq i<j \leq k}\left|\rho_{i j}\right| \exp \left(-\frac{u^{2}}{1+\left|\rho_{i j}\right|}\right) \leq c k^{-\delta_{0}},
$$

where $\delta_{0}=\{\nu(1-\delta)-\eta(1+\delta+\nu)\} /\{(1+\nu)(1+\delta)\}>0$ and $c$ is a constant independent of $n$ and $u$.

Proof of Theorem 1.3. (1.6) is obvious when $r=0$. In what follows, we assume that $0<r \leq \infty$. For $\theta>1$, let

$$
A_{k, l}=\left\{n: \theta^{k-1} \leq n \leq \theta^{k}, \theta^{l-1} \leq a_{n} \leq \theta^{l}\right\},
$$

where $k=1,2, \ldots ; l=1,2, \ldots$. The condition (vii)' implies that, for sufficiently large $k$, there exists $\gamma>0$ such that

$$
1 \leq l \leq k+1-\gamma \log ((k-1) \log \theta) /(\log \theta)^{2}=: K
$$

and there exists $M>0$ such that

$$
\theta(k, l):=\left[\theta^{k-l-1} / M\right]>1 .
$$


Noting that

$$
\lim _{n \rightarrow \infty} \frac{\sqrt{2 \log \left(n / a_{n}\right)}}{\beta_{n}^{\prime}}= \begin{cases}(r /(1+r))^{1 / 2} & \text { if } 0<r<\infty \\ 1 & \text { if } r=\infty\end{cases}
$$

by $\left(\right.$ vii) ${ }^{\prime}$, then (1.6) is proved if we show that

$$
\liminf _{n \rightarrow \infty} \sup _{0 \leq i \leq n} \frac{\left|S_{i+a_{n}}-S_{i}\right|}{\sigma\left(a_{n}\right) \sqrt{2 \log \left(n / a_{n}\right)}} \geq 1 \quad \text { a.s. }
$$

By the regular variation of $\sigma(\cdot)$, we have

$$
\sigma\left(\theta^{l-1}\right) \geq(\theta-1)^{-\alpha} \sigma\left(\theta^{l}-\theta^{l-1}\right)
$$

for some $0<\alpha<1$. Thus

$$
\begin{aligned}
& \liminf _{n \rightarrow \infty} \sup _{0 \leq i \leq n} \frac{\left|S_{i+a_{n}}-S_{i}\right|}{\sigma\left(a_{n}\right) \sqrt{2 \log \left(n / a_{n}\right)}} \\
\geq & \liminf _{k \rightarrow \infty} \inf _{1 \leq l \leq K} \inf _{n \in A_{k, l}} \sup _{0 \leq i \leq n} \frac{\left|S_{i+a_{n}}-S_{i}\right|}{\sigma\left(a_{n}\right) \sqrt{2 \log \left(n / a_{n}\right)}} \\
\geq & \liminf _{k \rightarrow \infty} \inf _{1 \leq l \leq K} \sup _{0 \leq i \leq \theta^{k-1}} \frac{\left|S_{i+\theta^{l}}-S_{i}\right|}{\sigma\left(\theta^{l}\right) \sqrt{2 \log \theta^{k-l}}} \\
& -\limsup _{k \rightarrow \infty} \inf _{1 \leq l \leq K} \sup _{0 \leq i \leq \theta^{k}} \sup _{\theta^{l-1} \leq j \leq \theta^{l}} \frac{(\theta-1)^{\alpha}\left|S_{i+j}-S_{i+\theta^{l}}\right|}{\sigma\left(\theta^{l}-\theta^{l-1}\right) \sqrt{2 \log \theta^{k-l}}} \\
= & : J_{1}-J_{2} .
\end{aligned}
$$

First, we will show that, for any small $\varepsilon>0$,

$$
J_{2} \leq \varepsilon \quad \text { a.s. }
$$

We claim that, for some $R>2$,

(2.7) $\limsup _{k \rightarrow \infty} \sup _{1 \leq l \leq K} \sup _{0 \leq i \leq \theta^{k}} \sup _{\theta^{l-1} \leq j \leq \theta^{l}} \frac{\left|S_{i+j}-S_{i+\theta^{l}}\right|}{\sigma\left(\theta^{l}-\theta^{l-1}\right) \sqrt{2 \log \theta^{k-l}}} \leq R \quad$ a.s.

By the same way as the proof of Lemma 2.1, we can obtain

$$
P\left\{\sup _{0 \leq i \leq \theta^{k}} \sup _{\theta^{l-1} \leq j \leq \theta^{l}} \frac{\left|S_{i+j}-S_{i+\theta^{l}}\right|}{\sigma\left(\theta^{l}-\theta^{l-1}\right)}>u\right\} \leq c_{\varepsilon} \theta^{k-l} e^{-u^{2} /(2+\varepsilon)}
$$


for all $u>1$. Thus,

$$
\begin{aligned}
& P\left\{\sup _{1 \leq l \leq K} \sup _{0 \leq i \leq \theta^{k}} \sup _{\theta^{l-1} \leq j \leq \theta^{l}} \frac{\left|S_{i+j}-S_{i+\theta^{l}}\right|}{\sigma\left(\theta^{l}-\theta^{l-1}\right)}>R \sqrt{2 \log \theta^{k-l}}\right\} \\
\leq & c_{\varepsilon} \sum_{l=1}^{K} \theta^{k-l} \exp \left(-\frac{8}{2+\varepsilon} \log \theta^{k-l}\right) \\
\leq & c_{\varepsilon} \sum_{l=1}^{K} \theta^{-2(k-l)} \leq c k^{-\gamma / \log \theta} .
\end{aligned}
$$

Since $\gamma / \log \theta>1$, the Borel-Cantelli lemma implies (2.7), and thus (2.6) follows if $\theta \rightarrow 1$.

Next, consider $J_{1}$. For $0 \leq m \leq \theta(k, l)$, let

$$
S(m)=S_{m \theta^{l} M+\theta^{l}}-S_{m \theta^{l} M} .
$$

It follows from (vii) that, for any $0<\varepsilon<1$,

$$
\begin{aligned}
& P\left\{\sup _{0 \leq i \leq \theta^{k-1}} \frac{S_{i+\theta^{l}}-S_{i}}{\sigma\left(\theta^{l}\right) \sqrt{2 \log \theta^{k-l}}} \leq \sqrt{1-\varepsilon}\right\} \\
& \quad \leq P\left\{\max _{0 \leq m \leq \theta(k, l)} \frac{S(m)}{\sigma\left(\theta^{l}\right)} \leq \sqrt{1-\varepsilon} \sqrt{2 \log \theta(k, l)}\right\} .
\end{aligned}
$$

Assume that (iv) holds. By Lemma 2.3, we have

$$
\begin{aligned}
& P\left\{\max _{0 \leq m \leq \theta(k, l)} \frac{S(m)}{\sigma\left(\theta^{l}\right)} \leq\{(2-2 \varepsilon) \log \theta(k, l)\}^{1 / 2}\right\} \\
\leq & \left(\Phi\left(\{(2-2 \varepsilon) \log \theta(k, l)\}^{1 / 2}\right)\right)^{\theta(k, l)} \leq \exp \left(-c\left(\theta^{k-l}\right)^{\varepsilon}\right),
\end{aligned}
$$

where $c$ is a positive constant. Hence by (2.8) and (2.9), we have

$$
\begin{aligned}
& P\left\{\inf _{1 \leq l \leq K} \sup _{0 \leq i \leq \theta^{k-1}} \frac{S_{i+\theta^{l}}-S_{i}}{\sigma\left(\theta^{l}\right) \sqrt{2 \log \theta^{k-l}}} \leq \sqrt{1-\varepsilon}\right\} \\
\leq & \sum_{l=1}^{K} \exp \left(-c\left(\theta^{k-l}\right)^{\varepsilon}\right) \leq \exp \left(-c k^{\varepsilon \gamma / \log \theta}\right)
\end{aligned}
$$

for all large $k$. It follows from the Borel-Cantelli lemma that

$$
J_{1} \geq 1 \quad \text { a.s. }
$$


Consider the case when (v) holds. In this case, we can estimate an upper bound of the right hand side of (2.8) by Lemmas 2.4 and 2.5 . Define

$$
r\left(m, m^{\prime}\right)=\operatorname{Cov}\left(\frac{S(m)}{\sigma\left(\theta^{l}\right)}, \frac{S\left(m^{\prime}\right)}{\sigma\left(\theta^{l}\right)}\right), \quad m>m^{\prime}=0,1, \ldots, \theta(k, l)
$$

and let $q=m-m^{\prime} \geq 1$. Then by (v) we have

$$
\begin{aligned}
& \left|r\left(m, m^{\prime}\right)\right| \\
= & \frac{1}{\sigma^{2}\left(\theta^{l}\right)} \mid E\left\{\xi_{m \theta^{l} M+1} \xi_{m^{\prime} \theta^{l} M+1}+\cdots+\xi_{m \theta^{l} M+1} \xi_{m^{\prime} \theta^{l} M+\theta^{l}}\right. \\
& \left.+\cdots+\xi_{m \theta^{l} M+\theta^{l}} \xi_{m^{\prime} \theta^{l} M+1}+\cdots+\xi_{m \theta^{l} M+\theta^{l}} \xi_{m^{\prime} \theta^{l} M+\theta^{l}}\right\} \mid \\
= & \frac{1}{\sigma^{2}\left(\theta^{l}\right)}\left|\rho_{q \theta^{l} M}+\cdots+\rho_{q \theta^{l} M+1-\theta^{l}}+\cdots+\rho_{q \theta^{l} M+\theta^{l}-1}+\cdots+\rho_{q \theta^{l} M}\right| \\
\leq & \frac{\left(\theta^{l}\right)^{2}}{\sigma^{2}\left(\theta^{l}\right)}\left|\rho_{q \theta^{l} M+1-\theta^{l}}\right| \leq \frac{\theta^{2 l}}{\sigma^{2}\left(\theta^{l}\right)} \frac{\sigma^{2}\left(q \theta^{l} M+1-\theta^{l}\right)}{\left(q \theta^{l} M+1-\theta^{l}\right)^{2}} \\
\leq & \frac{1}{(q M-1)^{2}} \frac{\sigma^{2}\left((q M-1) \theta^{l}\right)}{\sigma^{2}\left(\theta^{l}\right)} \leq c(q M-1)^{2 \alpha-2}<q^{-\nu},
\end{aligned}
$$

where $\nu=1-\alpha>0$. Applying Lemmas 2.4 and 2.5 for

$$
\begin{aligned}
& \xi_{l j}=\frac{S(m)}{\sigma\left(\theta^{l}\right)}, \quad m=0,1, \ldots, \theta(k, l), \\
& u=\{(2-\eta) \log \theta(k, l)\}^{1 / 2}, \quad \eta=2 \varepsilon \\
& \left|\rho_{i j}\right|=\left|r\left(m, m^{\prime}\right)\right|<\left|m-m^{\prime}\right|^{-\nu}, \quad m \neq m^{\prime}
\end{aligned}
$$

then the right hand side of (2.8) is less than or equal to

$$
(\Phi(u))^{\theta(k, l)}+c(\theta(k, l))^{-\delta_{0}}
$$

Thus we have

$$
\begin{aligned}
& P\left\{\sup _{0 \leq i \leq \theta^{k-1}} \frac{S_{i+\theta^{l}}-S_{i}}{\sigma\left(\theta^{l}\right) \sqrt{2 \log \theta^{k-l}}} \leq \sqrt{1-\varepsilon}\right\} \\
\leq & \exp \left(-c\left(\theta^{k-l}\right)^{\varepsilon}\right)+c\left(\theta^{k-l}\right)^{-\delta_{0}} \leq c\left(\theta^{k-l}\right)^{-\delta_{0}}
\end{aligned}
$$


for all large $k$. Considering $J_{1}$ in (2.5), we have

$$
\begin{gathered}
P\left\{\inf _{1 \leq l \leq K} \sup _{0 \leq i \leq \theta^{k-1}} \frac{S_{i+\theta^{l}}-S_{i}}{\sigma\left(\theta^{l}\right) \sqrt{2 \log \theta^{k-l}}} \leq \sqrt{1-\varepsilon}\right\} \\
\leq \sum_{l=1}^{K} c\left(\theta^{k-l}\right)^{-\delta_{0}} \leq c k^{-\gamma \delta_{0} / \log \theta} .
\end{gathered}
$$

Thus the Borel-Cantelli lemma gives (2.10). From (2.5), (2.6) and (2.10), we obtain (2.4). This completes the proof of Theorem 1.3.

ACKNOWLEDGEMENT. The authors wish to thank the referee for careful reading and helpful suggestion on this paper.

\section{References}

[1] S. A. Book and T. R. Shore, On large intervals in the Csörgö-Révész theorem on increments of a Wiener process, Z. Wahrsch. Verw. Gebiete 46 (1978), 1-11.

[2] Y. K. Choi, Erdös-Rényi-type laws applied to Gaussian processes, J. Math. Kyoto Univ. 31(1) (1991), 191-217.

[3] Y. K. Choi, Z. Y. Lin, W. S. Wang, K. S. Hwang and T. S. Kim, Asymptotic behaviors for partial sum processes of a Gaussian sequence, Acta Math. Hungar. 103(1-2) (2004), 43-54.

[4] E. Csáki and K. Gonchigdanzan, Almost sure limit theorems for the maximum of stationary Gaussian sequences, Statist. Probab. Lett. 58 (2002), 195-203.

[5] M. Csörgö, A glimpse of Pál Erdös's impact on probability and statistics, Canad. J. Statist. 30(4) (2002).

[6] M. Csörgö and P. Révész, Strong Approximations in Probability and Statistics, Academic Press, New York, 1981.

[7] M. Csörgő and J. Steinebach, Improved Erdös-Rényi and strong approximation laws for increments of partial sums, Ann. Probab. 9(6) (1981), 988-996.

[8] P. Deheuvels and J. Steinebach, Exact convergence rates in strong approximation laws for large increments of partial sums, Probab. Theory Related Fields 76 (1987), 369-393.

[9] A. N. Frolov, On one-sided strong laws for large increments of sums, Statist. Probab. Lett. 37 (1998), 155-165.

[10] L. Horvàth and Q. M. Shao, Darling-Erdös-type theorems for sums of Gaussian variables with long-range dependence, Stochastic Process. Appl. 63 (1996), 117137.

[11] H. Lanzinger and U. Stadtmüller, Maxima of increments of partial sums for certain subexponential distributions, Stochastic Process. Appl. 86 (2000), 307322.

[12] M. R. Leadbetter, G. Lindgren and H. Rootzen, Extremes and Related Properties of Random Sequences and Processes, Springer-Verlag, New York, 1983. 
[13] W. V. Li and Q. M. Shao, A normal comparison inequality and its applications, Probab. Theory Related Fields 122 (2002), 494-508.

[14] Z. Y. Lin, Path properties of Gaussian processes, Zhejiang University Press, 2001.

[15] — On Csörgö-Révész's increments of sums of non-i.i.d. random variables, Scientia Sinica (Series A) 30 (1987), no. 9, 921-931.

[16] _ On increments of sums of independent non-identically distributed random variables, Scientia Sinica (Series A) 31 (1988), no. 8, 927-937.

[17] Z. Y. Lin, Y. K. Choi and K. S. Hwang, Some limit theorems on the increments of a multi-parameter fractional Brownian motion, Stochastic Anal. Appl. 19 (2001), no. 4, 499-517.

[18] Z. Y. Lin and Q. M. Shao, On the limiting behaviors of increments of sums of random variables without moment conditions, Chinese Ann. Math. 14B (1993), no. $3,307-318$.

[19] J. Steinebach, On the increments of partial sum processes with multidimensional indices, Z. Wahrsch. Verw. Gebiete 63 (1983), 59-70.

[20] __ Improved Erdös-Rényi and strong approximation laws for increments of renewal processes, Ann. Probab. 14 (1986), no. 2, 547-559.

[21] W. S. Wang, Self-normalized lag increments of partial sums, Statist. Probab. Lett. 58 (2002), 41-51.

Yong-Kab Choi, Kyo-Shin Hwang, and Hee-Jin Moon

Department of Mathematics, College of Natural Science

Gyeongsang National University

Chinju 660-701, Korea

E-mail: math-ykc@hanmail.net

Tae-Sung Kim and Jong-Il Baek

Department of Mathematics and Institute of Basic Science

Wonkwang University,

Iksan 570-749, Korea

E-mail: starkim@wonkwang.ac.kr 